\title{
Weight loss and blood pressure normalization: the relevance of early interventions in hypertension
}

\author{
Arrigo FG Cicero \\ Hypertension Research (2010) 33, 195-196; doi:10.1038/hr.2009.227; published online 15 January 2010
}

$\mathrm{O}$ verweight and obesity are very wellknown risk factors for incident hypertension ${ }^{1}$ and for all-cause mortality; a recent estimate by US researchers suggests that obesity is per se responsible for one out of every 10 preventable deaths in the United States. ${ }^{2}$

Even though the most relevant guidelines in the field of cardiovascular disease prevention strongly emphasize the need for overweight and obese individuals to change their lifestyle to reduce body weight and thereby improve blood pressure and lower cardiovascular disease risk, ${ }^{3}$ there is a lack of direct evidence that this approach is useful in general practice. In fact, although potentially useful in the rigid setting of a clinical trial, at least one unfocused intervention did not significantly improve the blood pressure in a population setting. ${ }^{4}$ Moreover, a recent systematic review concluded that the available evidence supports the hypothesis that loss of $1 \mathrm{~kg}$ body weight corresponds to a decrease of $1 \mathrm{~mm} \mathrm{Hg}$ in systolic blood pressure, but only for follow-up periods of 2-3 years, possibly reflecting the fact that blood pressure often reverts to higher levels, regardless of whether weight loss is maintained. Moreover, this relationship is not clear with respect to diastolic blood pressure. ${ }^{5}$

In this context, the paper by Fogari et al. ${ }^{6}$ addresses a relevant issue. The authors evaluated the effect of weight loss on blood pressure (and blood pressure-related parameters) in a large sample of overweight mildly hypertensive patients. The patients who normalized their body mass index also experienced a larger improvement in blood

Dr AFG Cicero is at the Hypertension Research Unit, Internal Medicine, Aging and Kidney Disease Department, University of Bologna, Via Albertoni 15, Bologna 40138, Italy.

E-mail: afgcicero@cardionet.it pressure and in a number of metabolic and hormonal parameters, including insulin sensitivity, plasma leptin and activation of the renin-angiotensin-aldosterone system. Even if the decrease in leptin level proportional to weight loss is a physiological response, it must be highlighted that leptin level is a strong independent predictor of both hypertension and cardiovascular disease in overweight individuals. ${ }^{7}$ On the other hand, an overweight patient with mild hypertension exhibits the typical symptoms of the true metabolic syndrome, in which there is no confounder, such as a single maximal metabolic or hemodynamic alteration, and which is widely prevalent in the general population. This effect could be of particular interest in the United States and other Western countries, where the number of young overweight individuals has increased rapidly over the past few decades. ${ }^{8}$

Therefore, Fogari et al. point out the possibility that the hypertension classified in nearly $90 \%$ of cases as 'essential' is actually caused by a moderate (but just as damaging) increase in body weight in approximately one-third of these individuals. Almost as dramatic is the observation that, in the setting of a clinical trial carried out in a highly specialized setting, more than one-third of the patients were unable to lose a significant amount of weight, and less than one-half of the remaining patients did not achieve a normal body weight. A similar result was observed in a smaller study recently carried out in a Mexican population sample. ${ }^{9}$ A relevant issue that remains unexplored in the study by Fogari et al. is whether the obtained results will be maintained in the long term, that is, whether the patients who maintain their normal weight will develop hypertension in the future. Even if these patients develop hypertension, they will have less exposure to hypertension as a risk factor and will require drug medication later in life compared with overweight individuals.

This study also addresses the potential usefulness of orlistat in protocols aimed at reducing blood pressure in overweight patients. It is already known that orlistat is useful in achieving blood pressure reduction in obese patients, proportional to the dose used, the weight loss obtained and the baseline body weight. ${ }^{10}$ In this study, the authors used low-to-moderate drug dosages, proportional to the patient's body weight, with a very high tolerability profile. In this context, orlistat seems to be more useful in improving patient compliance with a reduced-calorie diet, and in reducing blood pressure than in accelerating weight loss.

On the basis of actual evidence, supported by the results of the study by Fogari et al., it seems very useful to intensify public health policies aimed at optimizing the body weight of the general population in order to reduce the incidence of hypertension and metabolic syndrome. The same effort must also be more intensively pursued in hypertension clinics and similar specialized settings, because a positive and relevant result is clearly achievable.

1 Forman JP, Stampfer MJ, Curhan GC. Diet and lifestyle risk factors associated with incident hypertension in women. JAMA 2009; 302: 401-411.

2 Danaei G, Ding EL, Mozaffarian D, Taylor B, Rehm J, Murray CJ, Ezzati M. The preventable causes of death in the United States: comparative risk assessment of dietary, lifestyle, and metabolic risk factors. PLoS Med 2009; 6: e1000058.

3 O'Keefe JH, Carter MD, Lavie CJ. Primary and secondary prevention of cardiovascular diseases: a practical evidence-based approach. Mayo Clin Proc 2009; 84: 741-757. 
4 Fodor GJ, McInnis NH, Helis E, Turton P, Leenen FH. Lifestyle changes and blood pressure control: a community-based cross-sectional survey (2006 Ontario Survey on the Prevalence and Control of Hypertension). J Clin Hypertens 2009; 11: 31-35.

5 Aucott L, Rothnie H, Mclntyre L, Thapa M, Waweru C, Gray D. Long-term weight loss from lifestyle intervention benefits blood pressure?: a systematic review. Hypertension 2009; 54: 756-762.

6 Fogari R, Zoppi A, Corradi C, Preti P, Mugellini A, Lazzari P, Derosa G. Effect of body weight loss and normalization on blood pressure in overweight non obese patients with stage 1 hypertension. Hypertension Research 2010; 33: 236-242.
7 Koh KK, Park SM, Quon MJ. Leptin and cardiovascular disease: response to therapeutic interventions. Circulation 2008; 117: 3238-3249.

8 Steinberger J, Daniels SR, Eckel RH, Hayman L, Lustig RH, McCrindle B, Mietus-Snyder ML, American Heart Association Atherosclerosis, Hypertension, and Obesity in the Young Committee of the Council on Cardiovascular Disease in the Young; Council on Cardiovascular Nursing; and Council on Nutrition, Physical Activity, and Metabolism. Progress and challenges in metabolic syndrome in children and adolescents: a scientific statement from the American Heart Association Atherosclerosis, Hypertension, and Obesity in the Young Committee of the Council on
Cardiovascular Disease in the Young; Council on Cardiovascular Nursing; and Council on Nutrition, Physical Activity, and Metabolism. Circulation 2009; 119: 628-647.

9 Márquez-Celedonio FG, Téxon-Fernández 0 , ChávezNegrete A, Hernández-López S, Marín-Rendón S, Berlín-Lascuraina S. Clinical effect of lifestyle modification on cardiovascular risk in prehypertensives: PREHIPER I study. Rev Esp Cardiol 2009; 62: 86-90.

10 Siebenhofer A, Horvath K, Jeitler K, Berghold A, Stich AK, Matyas E, Pignitter N, Siering U. Long-term effects of weight-reducing drugs in hypertensive patients. Cochrane Database Syst Rev 2009; Art. No. CD007654. 